\title{
Spectroscopic Study of Electronically - Doped Polyanilines
}

\author{
Hitesh Parmar ${ }^{1}$, R.K.Shah ${ }^{1}$, Vishal.R.Jain ${ }^{2}$, H.S. Patel ${ }^{3}$, Ketan Dodiya ${ }^{4}$, A.T.Oza ${ }^{4}$ \\ ${ }^{1}$ Arts,Science and R.A. Patel Commerce College Bhadran, Gujarat, India. \\ ${ }^{2}$ Navjevan Science Collegxe, Dahod, Gujarat,India. \\ ${ }^{3}$ Arts, Commerce and Science College, Borsad, Gujarat,India. \\ ${ }^{4}$ Department of Physics, SPU, Vallabh Vidyanagar, Gujarat, India
}

\begin{abstract}
Polyanilines namely emeraldine, nigraniline and pernigraniline were prepared by chemical method and were electronically-doped with organic acceptors such as, TCNE, TCNQ, DDQ, Chloranil and KI-I2. The FTIR spectra of $20 \%$ and $50 \%$ doped polyanilines were obtained and analyzed using small polaron model which connected electrical conductivity with IR absorption. Emeraldine prepared by standard method and pure emeraldine as readily obtained wee used. The absorption profiles as experimentally obtained and as calculated using small-polaron model were compared. This comparison leads to frequency -dependence of the real part of refractive index which shows dispersion shape as desired.
\end{abstract}

Keywords: Infrared spectra, polaron

PACS: 78-Optical properties, condensed-matter spectroscopy, and other interactions of radiation and particles with condensed matter

\section{INTRODUCTION:}

Polyaniline polymers have been studied by many solid state physicists (1-6). Magnetic $(7,8)$,optical $(7,9)$,photo-induced optical(10-12) and transport (1318) properties have been studied in details for polyaniline namely emeraldine. There are two types of doping (1) protonic doping and (2) electronic doping. Protonic doping is carried out by doping various acids such as sulphonic acid (19), camphor-sulphonic acid (20), dibenzoyl tartaric acid (21), hetero-poly acid (22), H2SO4 (23), 2-acrylamido-2-methyl-1-propane sulfonic acid (AMPSA), Lewic acids (25) and sulphosalicylic,salicylic and citric acids (26).
Electronic doping is done rarely. Therefore, in the present study electronically-doped polyanilines using organic electron-acceptors such as TCNE, TCNQ, DDQ, Chloranil and KI-I2 have been prepared and studied using FTIR spectroscopy.

\section{EXPERIMENTAL PROCEDURE}

Emeraldine (SM) was prepared by standard method using ammonium peroxy disulfate synthesis (27). Recently we standardized method of preparing other firms of polyanilines namely protoemeraldine, nigraniline and pernigraniline (28). Emeraldine (pure) was obtained from standard chemical company of USA molecular structures of polyanilines are shown (figure 1). Both forms of emeraldine (SM and Pure) wewe doped with TCNE, (tetracyanoethylene), TCNQ(7,7,8,8 - teracyano-p-quinodimethane), DDQ (2,3-dichloro-5,6-dicyano-p-benzoquinone), Chloranil and KI-I2 in $20 \%$ and50\%. It was found that emeraldine prepared by standard method was lessoxidized than emeraldine (pure) obtained from chemical company. Emeraldine (pure) was nearer to nigraniline while emeraldine $(\mathrm{sm})$ was nearer to protoemeraldine. This was concluded on the basis of effect of doping increasing absorption in emeraldine (sm) and decreasing absorption in emeraldine (pure) with any acceptor.

Protoemeraldine(brown), emeraldine (green), nigraniline(blue) and pernigraniline (violet)were mixed with organic accrptors such as TCNE, TCNQ, DDQ ,Chloranil and KI-I2 and grinded in a morter with a pastle till colour changed. Further these fine homogeneous powder were mixed with dry 
spectrograde $\mathrm{KBr}$ powder and again grinded. Palates in the form of circular discs were prepared from these mixtures with a manally operated compressing machine. These discs were placed in a dark chamber of GXFTIR single beam spectrophotometer fabricated by Perkin Elmer Company, USA.

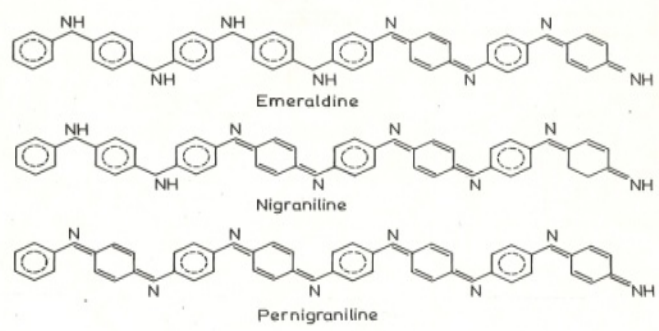

(fig. 1)

\section{RESULTS AND DISCUSSION}

Low - mobility material cannot be understood within the framework of the usual band piture and therefore, small polarons due to strong electron-phonon coupling are found to be responsible for transport properties. The dc hopping conductivity of small polarons in crystals with the resonance integrals only between the nearest-neighbours is given by

$$
\sigma_{h}=e \mu_{0} n(1-f) \frac{\hbar \omega}{K_{B} T}
$$

Where $f=\left(e^{-\beta \mu}+1\right)^{-1}$ Is related with occupation of a site, $n=\frac{N f}{\Omega}$ is the concentration of the charge carriers and $\mu_{0}=e a^{2} / \hbar$ has dimension of mobility. $\mathrm{W}$ is the hopping probability. Under certain approximation, the d.c. conductivity takes the form (29).

$\sigma_{h}=e \mu_{0} n(1-f) \frac{\sqrt{\pi}}{2} \frac{J^{2}}{K_{B} T \sqrt{E_{a} K_{B} T}} \exp \left(-\frac{E_{a}}{K_{B} T}\right)$

For frequency dependence $0 \mathrm{f}$ conductivity

$\varepsilon(\omega)=\bar{\varepsilon}(\omega)+\frac{4 \pi i \sigma(\omega)}{\omega}$

Where $\bar{\varepsilon}(\omega)$ denotes contribution to dielectric constant $\varepsilon(\omega)$ resulting from lattice mechanism. Then $\sigma(\omega)$ is also complex and real and imaginary parts $\sigma^{\prime}(\omega)$ and $\sigma^{\prime}(\omega)$ are given by

$\sigma^{\prime}(\omega)=\sigma_{h} \frac{\sinh \left(\frac{\hbar \omega}{2 K_{B} T}\right)}{\frac{\hbar \omega}{2 K_{B} T}} \exp \left(-\frac{1}{4} \omega^{2}(\delta t)^{2}\right)$

And $\sigma_{h}$ is the d.c. hopping conductivity. For high temperatures,

$K_{B} T \gg \hbar \omega_{0} p / 2, \quad \delta t=\hbar / \sqrt{4 E_{a} K_{B}} T$,

$\sigma^{\prime}(\omega)$ is given by

$$
\begin{aligned}
& \sigma^{\prime}(\omega) \\
& =\left[e \mu_{0} n(1-f) \frac{\sqrt{\pi}}{2} \frac{J^{2}}{\sqrt{E_{a} K_{B} T}}\right] \\
& \times \frac{1-\exp \left(-\frac{\hbar \omega}{K_{B} T}\right)}{\hbar \omega} \exp \left(-\frac{\left(\hbar \omega-4 E_{a}\right)^{2}}{16 E_{a} K_{B} T}\right)
\end{aligned}
$$


$\mathrm{J}$ is exchange integral and $\mathrm{E}_{\mathrm{a}}$ is activation energy. Thus, the dependence $\sigma^{\prime}(\omega)$ he form of a weakly asymmetric gaussian which has peak centered about $\hbar \omega=4 E_{a}$ and having half-width $4 \sqrt{E_{a} K_{B} T}(32,33)$. Now the absorption coefficient $\alpha$ is related with $\sigma^{\prime}(\omega)$ according to

$$
\begin{aligned}
& \sigma^{\prime}=\frac{\alpha \mathrm{n}_{1} \mathrm{c}}{4 \pi} \\
& \alpha=\left(\frac{4 \pi}{\mathrm{n}_{1} \mathrm{c}}\right) \sigma^{\prime}(\omega)
\end{aligned}
$$

The above small - polaron model is applied to optical properties of doped-polyanilines since the FIIR spectra of all electronically-doped polyanilines contain a big (intenses and broad asymmetric gaussian band in the range between $1600 \mathrm{~cm}^{-1}$ and $4000 \mathrm{~cm}^{-1}$.

The protoemeraldine FIIR spectra of $20 \%$ electronically-doped these spectra contain asymmetric Gaussian bands in the range between $2400 \mathrm{~cm}^{-1}$ and $3800 \mathrm{~cm}^{-1}$.

For $\hbar \omega=4 E_{a}$

$\sigma_{\max }^{\prime}(\omega)=\left[e \mu_{0} n(1-f) \frac{\sqrt{\pi}}{2} \frac{J^{2}}{\sqrt{E_{a} K_{B} T}}\right]\left(\frac{1-e^{\frac{-4 E_{a}}{K_{B} T}}}{4 E_{a}}\right)$

$\mathrm{K}=$ constant

$$
\alpha_{\max }=\left(\frac{4 \pi}{n_{1} c}\right) \sigma_{\max }^{\prime}
$$

The theoretical Gaussians were calculated using the equation 5 and $\mathrm{C}$ was calculated using

$K=C \times\left(\frac{1-e^{\frac{-4 E_{a}}{K_{B} T}}}{4 E_{a}}\right)$

These gaussians are plotted (Figure 2).

$\frac{\alpha_{T}(\omega)}{\alpha_{E}(\omega)}=\frac{n_{1}(\omega)}{n_{1}\left(\frac{4 E_{a}}{\hbar}\right)}$

Since $\alpha_{\mathrm{T}}(\omega)$ assumes $\mathrm{n}_{1}$ as a constant but actual $\alpha_{\mathrm{E}}(\omega)$ experimental curves contains $\mathrm{n}_{1}$ which depends on frequency. The asymmetry of $\alpha_{\mathrm{T}}(\omega)$ is contained in $\frac{1-e^{\frac{-4 E_{a}}{K_{B} T}}}{\hbar \omega}$ factor only while the asymmetry of $\alpha_{\mathrm{E}}(\omega)$ also contains the factor $1 / \mathrm{n}_{1}(\omega)$. For protoemeraldine type polyaniline, $4 \mathrm{E}_{\mathrm{a}} / \hbar=3200-3300 \mathrm{~cm}^{-1}$, and hence $E_{a} \cong 0.1 \mathrm{eV}$. However, the half-width gives somewhat lesser value of $E_{a}$.

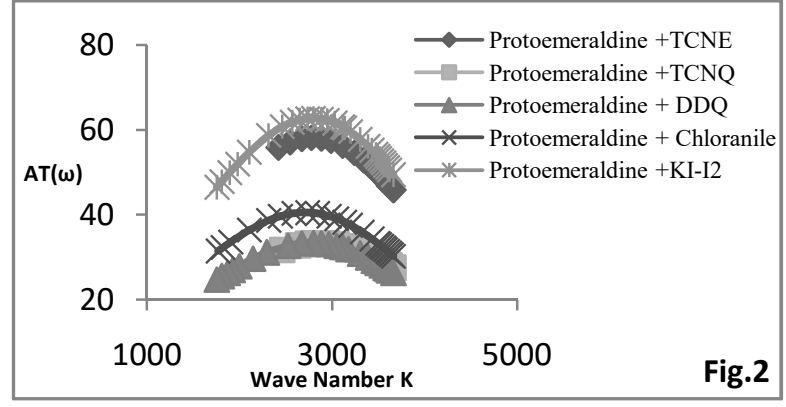

Protoemeraldine itself has four gaussians in the infrared range which is consistent with its resonance structure. Two Gaussians seem to be merged to form a large and broad asymmetric Gaussian peak as analyzed above in the spectra of electronicallydoped protoemeraldine. Two Gaussian peaks remain intact around $1590 \mathrm{~cm}^{-1}$ and $900 \mathrm{~cm}^{-1}$ with some distortions. The high energy (Frequency) Gaussian covering all the benzenoid and quinonoid rings only get modified by the charge transfer to the acceptor. Thus in electronically - doped polyaniline intermolecular interactions among large oligoaniline molecules are governed by negatively charged acceptor molecules.

Similar is the case of emeraldine (sm) prepared by standard method. A board asymmetric Gaussian band is the characteristic feature of all of the FTIR spectra of CT complexes of emeraldine The highest frequency Gaussian band associated with all benzenoid and quinonoid rings is modified into an asymmetric Gaussian associated with hopping of polarons among the oligoaniline molecules. Here $E_{a}=0.07 \mathrm{ev}$ and $E_{a}$ found from the band-width is $\mathrm{E}_{\mathrm{a}}=0.06 \mathrm{ev}$. Other two out of three Gaussian bands are found at $1150 \mathrm{~cm}^{-1}$ and $600 \mathrm{~cm}^{-1}$ at lower frequencies and remain intact apart from slight distortion $\alpha_{\mathrm{T}}(\omega)$ which are calculated and fitted are shown.(fig. 3).

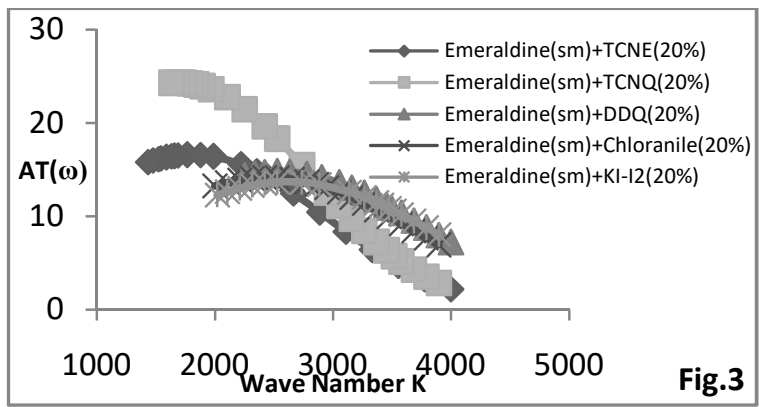

The FTIR spectra of CTCS of emeraldine (pure) (emeraldine obtained from chemical company) are also shown. The calculated asymmetric Gaussian curves are also shown (fig. 4). 


\section{www.ijtsrd.com}

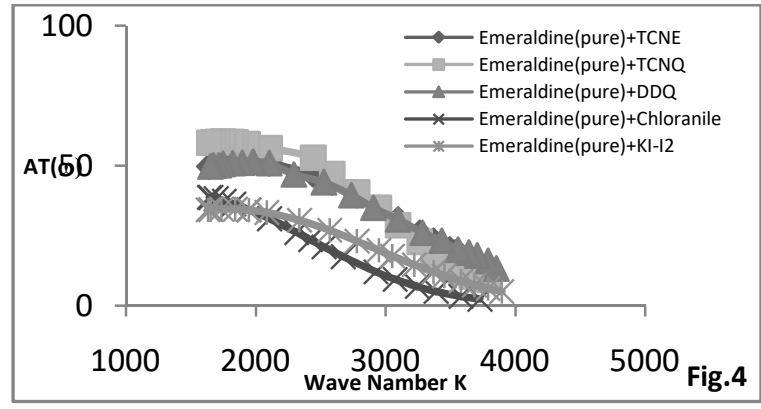

Emeraldine availed from a chemical method was more oxidized than emeraldine prepared by standard method. Thus emeraldine was more near nigraniline as obtained from a company while emeraldine was more near protoemeraldine as prepared by standard method. The maximum absorption decreased by electronic doping in the case of pure emeraldine while maximum absorption increased by doping the emeraldine prepared by standard method. Electronic doping has effect of deprotonation. Emeraldine has the highest conductivity or absorption among all the polyaniline.

Nigraniline was also $20 \%$ doped with various acceptors and FTIR spectra of CTCS of nigraniline are obtain these spectra also contain asymmetric Gaussian band which can be described within small-polaron model. These asymmetric Gaussian bands are theoretically fitted (fig. 5).

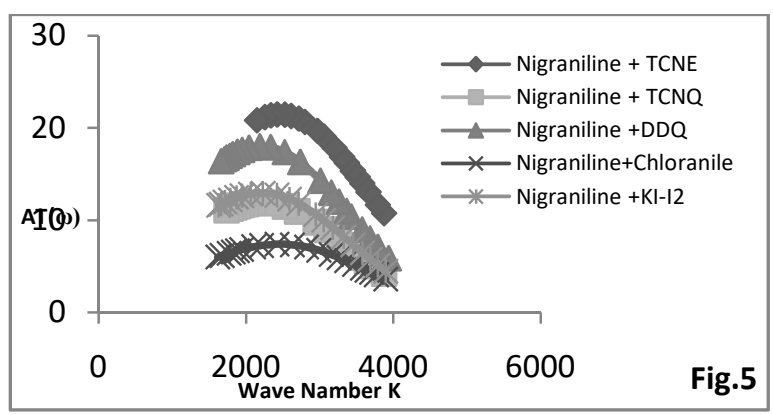

The ratio of $\alpha_{\mathrm{T}}(\mathrm{w}) / \alpha_{\mathrm{E}}(\mathrm{w})$ again gives frequency dependence of refractive index $n_{1}(w)$. there is one more Gaussian band around $1130 \mathrm{~cm}^{-1}$ which is somewhat distorted.

The Gaussian band in CTCS of pernigraniline is again sharp similar to those in protoemeraldine cases. The Gaussian again centered around higher frequency (about $3200 \mathrm{~cm}^{-1}$ ) as compared to the emeraldine is sharp and having band tailing towards low frequency side. This Gaussian is also theoretically fitted (fig. 5). The taling towards low frequency side can be analyzed as nature of transition between valence and conduction band since pernigraniline and its CTCS are purely semiconductors. The spin-orbit splitting of valence band and consequent three transitions from valence sub bands to conduction band are also evident in all of the CTCS of pernigraniline.

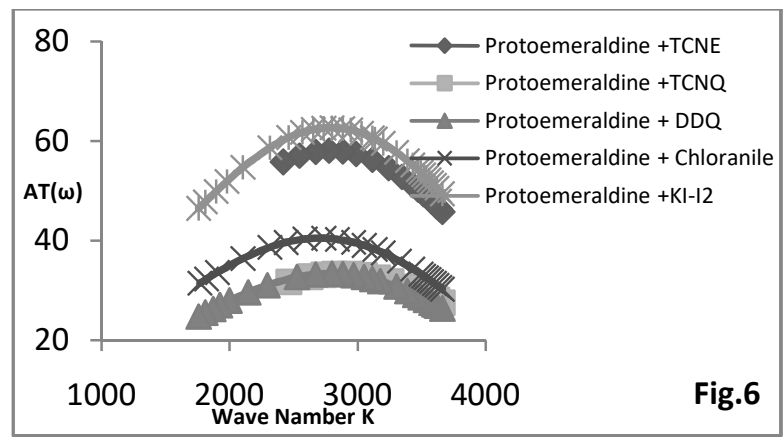

Now this follows discussion of 50\% doping of emeraldine (SM) and emeraldine(pure) with organic acceptors. As expected, maximum absorption of asymmetric Gaussian is increased by $10-15 \%$ as compared to $20 \%$ doping. This means that CTCS with more dopant concentration increases electrical conductivities. The FTIR spectra of CTCS of emeraldine (SM) + DDQ complex is an exception where no gaussian band is found in high frequency range rather a nature of transition obeying $A h \vartheta=B\left(h \vartheta-E_{g}\right) \quad$ is found (fig. 7).

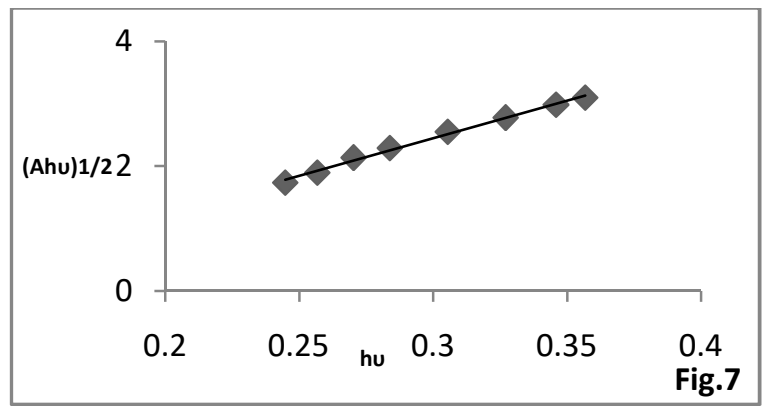

This shows macromolecular behavior of emeraldine because it is an allowed indirect transition. The FTIR spectra of emeraldine (pure) doped with organic acceptor. Theoretically fitted asymmetric Gaussian bands are also shown

(fig.8). 


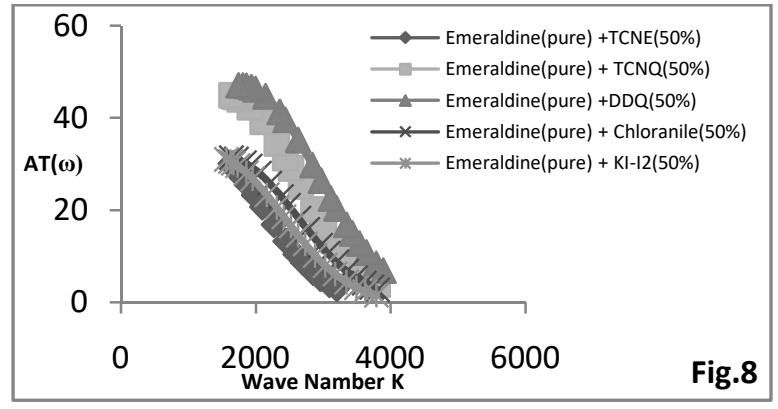

the Gaussian bands in these cases are very broad spanning the entire infrared range. The two more Gaussian bands of emeraldine at low frequencies are merged strongly overlapping each other. Here $E_{a}$ calculated with absorption maximum at $\hbar \omega=4 E_{a}$ is less than $\mathrm{E}_{\mathrm{a}}$ calculated using band-width because band width is found to be very large.

\section{CONCLUSION}

The electronically-doped polyanilines contain a broad and intense asymmetric Gaussian in the infrared spectra associated with optical properties in small-polaron model. Thus polarons are generated along the chains of polyanilines due to electronic doping. Electronic doping with organic acceptors leads to an enhancement of electrical conductivity which increases with dopant concentration. For the first time different types of polyanilines namely protoemeraldine, emeraldine, nigraniline and pernigraniline are comparatively studied along with their polymeric CTCS with organic acceptors.

\section{REFERENCES}

1. H.H.S.Javedi,K.R.Kromack,A.G.MacDiamird,andA.J.Epstein ,Phys.Rew.B,39,3579,1989.

2. J.C.Chiang and A.J.MacDiamird, Synth. Metals, 13,193,1986.

3. A.G.MacDiamird, J.C.Chiang, A.F.Ritcher and A.J.Epstein, syn. metals, 18,285,1987.

4. J.F.Trovers ,J.Chrocozek, F.Devereux, F.Granoud, M. Neehitchein,A.syed,E.M.Genies and c.sintavis, mol.crystLiq. Cryst.,121,195,1985.

5. A.G.MacDiamird,R.J.mammong,S.L.Mu,N.L.D.Somasiri, and w.wu, polym.prepr,25,248, 1984 .
6. A.G. MacDiamird,J.C. chiang, M. Helprin, W.S.Huang, S.L.mu,N.L.D.Samasiri, W.Wu and S.I.Yaniger, MOL Cryst Liq.Cryst, 121,173,1985.

7. A.J.Epstein, J.M.Ginder,F.Zuo,R.W.Bigclow, H.S.Won D.D.Tanner, A.F.Richter,W.S.Huang, and A.G.MacDiamird ,synth.metals ,18,303,1987.

8. J.M.Ginder,A.F.Ritcher,A.G.macDiamird,and A.J,Epstein, solid state commun,63,97,1987.

9. H.Huzmany,N.S.Sariciflei, H. neuger-bauer,and A.Neekei,Phys.Ren Letters,60,212,1988.

10. M.G.Roz,J.M.Ginder,F.E.Wigen,A.J.Epstain,M.Angelopoulo s, and A.G. MacDiamird,PhysRev,Lett.60,2789,1988.

11. A.J.Epstein,J.M.Ginder,M.G.Roe,J.L.Gustafson,M.Angelopo ulos and A.G.macDiamird, in materials Research conference proceedings, ed. By A.J. Heeger,J.Grenstein and D.R.Ulrich,MRS Pittsburg 1988,vol.109,p-313.

12. R.P.McCall,M.G.Roe,J.M.Ginder,T.Husumoto,A.J.Epstein, E.M.Scherr and A.G.macDiamird, Synth.Mct.,29,433,1989.

13. F.Zuo, M.Angeloprulos,A.G.MacDiamird and A.J.Epstein, phys-Rev,B36,3475,1987.

14. H.H.S.Javedi, F.Zuo,M.Angelopoulos,A.G.MacDiamird and A.J.Epstein,Mol.Cryst. Liq.Cryst,160,225,1988.

15. T.Hayashi,Y.Hirav,H.Tanaha and T.Mistri,Jpn.J.App phys, 26,1800,1987.

16. H.H.S.Javedi, M.Angelopoulis, A.G.MacDiamird and A.J.Epstein, Synth Met., 26,1,1988.

17. B.Lundberg,W.R.Salanech, and J.Lundstrom, Syngh met.,24,143,1987.

18. F.Zuo, M.Angelopoulos, A.G.MacDiamird and A.J.Epstein, Phys-Rev B39,3570,1989.

19. S.F.S.Draman, R.Daik, and A.Musa, world academy of science, Engineering and Technology, 43,464,2008.

20. F.Gervsis, N.petit, C.Popon and P.Buvat, Eur. Phys. J.B 31,47,2003.

21. Macera Naar, Saad Lamouri, Belhacem Belaabcd, Tojik Houroghti and Mourdine Gabouze, poly.J.L. 41,432,2009.

22. Jian Gong, Xiu Jun Cui, shou Guv Wang, Zhong Wei Xie and Lun $\mathrm{Yu} \mathrm{Qu}$, Chinese chemical Letters, 13,123,2002.

23. P.D.Gaihwad, D.J.Shirale, V.K.Gade, P.S.Savale, H.J.Hharat, K.P. HaHde, S.S.Hussaini, N.R. Dhumane and M.D.shirsat,Bull mater Sci, 29,169,2006.

24. V.Sitaram, Ajay Sharma, S.V.Bhat, K.Mizoguchi and Raghu menon A preprint.

25. Krzyslof BienKowski, Jean-Louis Oddou, Olivier Horner, Irena Kulszewicz-Bajer, Francoise Genoud, JanSuwalski and Adam pron, Nucleonika, 48,53,2003.

26. Farideh Ghadimi, Kazem Dindar safa, Bakhashali Massoumi and Ali Akber Entezami, Iranian Polymer Journal, $11,159,2002$.

27. H.Bottger and V.V.Bryksin, Hopping conduction in solids , VCH Weinheim FL, 1985 (P. 55).

28. M.I.Klinger, Phys.Letters, 7,67,102,1963.

29. H.G.Reik, solid state commun, 1,67,1963. 